Rev. High Pressure Sci. Technol., Vol. 7 (1998) 142 144

\title{
Simultaneous Gigahertz Ultrasonic Interferometry and X-ray Diffraction in a New Diamond Anvil Cell
}

\author{
W. A. Bassett ${ }^{1}$, H. Spetzler ${ }^{2}$, R. J. Angel ${ }^{3}$, G.R. Chen ${ }^{4}$, A. H. Shen ${ }^{5}$, H. -J. Reichmann ${ }^{3}$, A. Yoneda ${ }^{6}$ \\ 1 Dept. Geol. Sci., Cornell University, Ithaca, NY 14853 \\ 2 Dept. Geol. Sci. \& CIRES, Univ.of Colorado, Boulder, CO 80309 \\ 3 Bayerisches Geoinstitut, Universitat Bayreuth, D-95440 Bayreuth, Germany \\ 4 Dept. Earth Space Sci., SUNY Stony Brook, Stony Brook, NY 11794-2100 \\ 5 Dept. of Earth Sciences, Downing Street, University of Cambridge, Cambridge, CB2 3EQ, UK; Current \\ address: Department of Earth Sciences, University of Cambridge, Downing Street, Cambridge, CB2 3EQ, England. \\ 6 Dept. of Earth and Planetary Sciences, Nagoya Univ., Nagoya, Japan
}

\begin{abstract}
Advances in the generation and transmission of very high frequency ultrasonic signals make it practical to measure elastic properties in single crystals small enough to be subjected to high pressures and temperatures in a new diamond anvil cell designed for simultaneous ultrasonic and x-ray diffraction measurements up to $5 \mathrm{GPa}$ and $400^{\circ} \mathrm{C}$. In addition to providing a new accurate method for measuring elastic properties this technique can provide simultaneous equations of state which can be used to produce a new primary pressure scale. Preliminary measurements on $\mathrm{MgO}$ show the feasibility of this new technique.
\end{abstract}

\section{Introduction}

Instrumentation capable of accurate measurements of elastic properties at high pressures and temperatures is an important source of data for interpretation of the Earth's interior. In addition, establishing a reliable pressure scale is of fundamental importance in all areas of high pressure research. At modest pressures the absolute pressure scale is based upon simple force over area measurements in a piston-cylinder type of apparatus $[1,2]$. Most current pressure calibrations greater than $1 \mathrm{GPa}$ are tied, directly or indirectly, to the theoretical calculation of the Equation of State for sodium chloride [3]. At higher pressures, EOS data for certain metals based upon shock measurements have been used $[4,5,6]$. All pressure scales for pressures higher than $1 \mathrm{GPa}$ require assumptions about the materials involved.

In this paper we describe instrumentation which allows us to make ultrasonic travel time measurements on a single crystal at high pressure and temperature in a diamond anvil cell at the same time that we make lattice parameter measurements on the same single crystal by $\mathrm{x}$-ray diffraction. These measurements yield, respectively, $\mathrm{K}_{\mathrm{S}}=-\mathrm{V}(\mathrm{dP} / \mathrm{dV})_{S}$ and $\mathrm{V}$ as a function of pressure and temperature. If these measurements are then made simultaneously over a range of pressure and temperature, an equation of state can be developed that is independent of any additional pressure measurement and can therefore be used as an independent pressure scale [7]. This approach is essentially the same as that of the very accurate measurements of the freezing point of mercury made by Ruoff et al. [8], although the authors of that study approximated the conversion of $\mathrm{K}_{\mathrm{S}}$ to $\mathrm{K}_{\mathrm{T}}$.

\section{Diamond Anvil Cell}

X-ray diffraction and ultrasonic measurements within a single diamond anvil cell require rather different modifications. We have solved the somewhat conflicting requirements by designing a new diamond anvil cell into which the ultrasonic signal is introduced through one of the diamond anvils while $x$-rays enter and exit through the sides. In this cell both measurements can be carried out to temperatures up to $400^{\circ} \mathrm{C}$ and pressures up to $5 \mathrm{GPa}$.

The diamond anvil cell is based upon a successful hydrothermal cell design [9] subsequently developed to allow $\mathrm{GHz}$ ultrasonic measurements $[10,11]$. In the new cell the sample is placed in a gasket between two brilliant-cut, gemquality, 1/8-carat diamonds mounted on tungsten carbide seats with axial holes that permit access to the sample for light and, in this case, the ultrasonic signal. Molybdenum wires wound around the tungsten carbide seats serve as heaters. The geometry of the carbide seats and the heater windings has been changed to increase the $\mathrm{x}$-ray access to the sample (Figure 1). Temperatures greater than $300-400^{\circ} \mathrm{C}$ will require further modifications to prevent oxidation of the anvils, supports, and heater windings. The two halves of the cell are connected by just two posts, the ends of which are threaded to take nuts that are used to pull together the top and bottom platens to generate pressure. This two-post system has the advantage of maximizing $\mathrm{x}$-ray access to the sample. For $\mathrm{x}$-ray diffraction measurements the cell is attached to a modified $x-y$ goniometer head by four ceramic posts (for heat insulation) and an intermediate steel plate to act as heat shield. Because this attachment is made via the lower platen of the cell, the buffer rod can be left in place during diffraction measurements, and ultrasonics measurements can be made while the cell is mounted on the $\mathrm{x}$-ray single-crystal diffractometer.

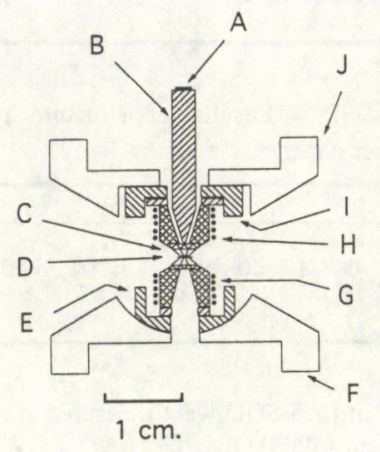

Fig. 1. Cross-section through the central portion of the new diamond anvil cell. A) transducer, B) buffer rod, C) upper diamond anvil, D) sample, E) alignment rocker, F) lower platen, G) lower heater, H) upper heater, I) alignment slider, J) upper platen. 


\section{3. $\mathrm{GHz}$ Ultrasonics}

Because the crystal samples in the diamond anvil cell are much thinner than samples typically used in conventional ultrasonic measurements, the frequency of the ultrasonic signals must be increased by the same factor to obtain short enough wavelengths to yield similar precision in measurement. This means attaining frequencies in the $\mathrm{GHz}$ range for $\mathrm{p}$-waves and because of their lower velocity, $500 \mathrm{MHz}-1 \mathrm{GHz}$ for s-waves. Frequencies of $2.4 \mathrm{GHz}$ have been obtained using transducers which we sputtered directly onto the sapphire buffer rod. We have employed the procedure of bonding and subsequently thinning $\mathrm{LiNbO}_{3}$ transducers mounted on the buffer rod for producing transverse waves with only limited success. We are now experimenting with the conversion of compressional waves to shear waves by scattering from surfaces cut at specific angles in the buffer rod or within the anvil itself.

Ultrasonic signals from the transducer are introduced to the sample by means of a single-crystal sapphire buffer rod pressed against the table face of the diamond anvil carrying the sample (Figure 1). Measurements of travel times of the ultrasonic signals in the sample are made by interfering the signal reflected from the far end of the sample with that reflected from the diamond/sample interface. The faces of the buffer rod as well as the faces of the diamond anvil must be cut and polished with a high precision. To this end we have developed a procedure using reflected light for cutting and polishing the culet and table faces of the diamonds parallel to within a few milliradians of arc. The mounting device that holds the buffer rod assembly to the diamond anvil cell allows it to be translated and tilted to bring its end into exact parallelism with the diamond table face.

\section{Operation}

The diamond anvil cell that we have described has been successfully pressure tested to approximately $4.4 \mathrm{GPa}$ with diamond anvils whose culet faces were $1 \mathrm{~mm}$ in diameter. Ultrasonic measurements, for which details of data collection, analysis, and reduction are the same as those described previously $[10,11]$, have been performed on samples of $\mathrm{MgO}$ and $\mathrm{CaF}_{2}$ ranging in thickness from 50 to $200 \mu \mathrm{m}$ using $\mathrm{KBr}$ or methanol/ethanol mixtures as pressure media.

In terms of angular access to the sample for $x$-rays this diamond anvil cell is very similar in concept to that described in detail by Koepke et al. [12]. The umbilical cord that connects the buffer rod to the electronic instrumentation for ultrasonic measurements places no limits upon diffractometer operation. X-rays pass at high angles to the load axis of the diamond anvil cell, and most diffraction occurs with the incident and diffracted beams passing through the same diamond anvil. The opening on each side of the cell provides an access angle of approximately $65^{\circ}$ in the plane perpendicular to the sample plane and $120^{\circ}$ within the sample plane. The gasket prevents any access to the sample by $\mathrm{x}$-rays within approximately $15^{\circ}$ of the sample plane. This geometry allows two or three reflections to be used for centering using the the eight positions required by Hamilton's method of diffracted beam centering [13]. Diffractometer setting angles for these reflections were obtained by the methods described in detail by Angel et al. [14] and are capable of a precision of about 1 part in 7,000 by vector least-squares fit [15]. These measurements agree within the uncertainties with the previously determined equation of state of $\mathrm{MgO}$ [16] (Figure 2).

\section{Results}

A MgO crystal attached to the upper diamond anvil with Super Glue and subjected to pressure at room temperature in an ethanol pressure medium yielded ultrasonic travel time data by the uitrasonic method and unit cell parameter data by the single crystal $x$-ray diffraction method. Figure 3 is a plot of travel

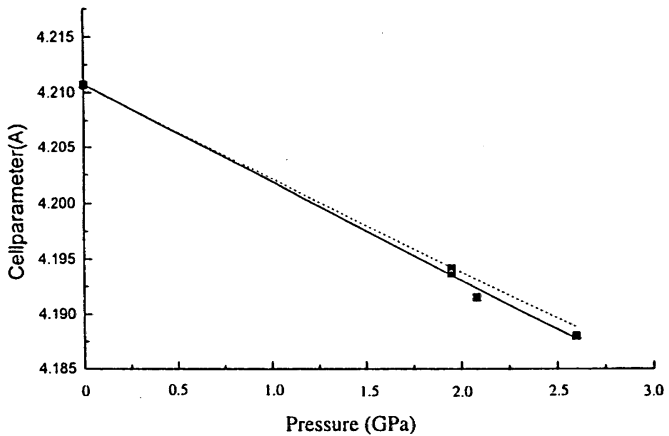

Fig. 2. Plot of unit cell parameter of $\mathrm{MgO}$ versus pressure measured by the ruby method. The solid squares are our data points. The solid line is fit to our data. The dashed line is from Jackson and Niesler [16]. The error is smaller than the solid squares.

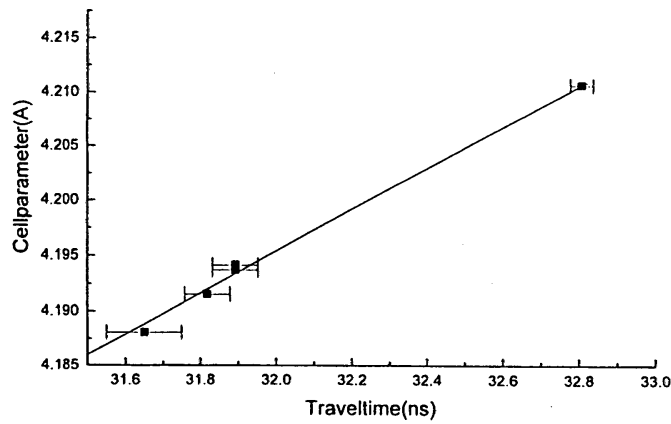

Fig. 3. Plot of the unit cel parameter of $\mathrm{MgO}$ versus traveltime of the ultrasonic signal along the [100] direction. The solid squares are our data points. The solid line is the fit to our data.

time versus unit cell parameter. Although we also measured pressure by the ruby method, we plot these parameters to show how small the scatter is. The pressure of the highest point, measured by the ruby method, is $2.6 \mathrm{GPa}$.

\section{Future Prospects}

The cell design that we have described has been successful in allowing the combination in one diamond-anvil pressure cell of the previously developed techniques of high-precision single-crystal $\mathrm{x}$-ray diffraction $[14,17]$ and $\mathrm{GHz}$ ultrasonic wave velocity measurements $[18,10]$. As in the previous cell design for ultrasonics measurements [10], the contact between the buffer rod and the diamond anvil has been accomplished with a normal force without the use of a bond, while the contact between the diamond anvil and the single crystal sample has utilized a bonding agent. Using hydrostatic fluid pressure media and glued bonds between sample and diamond anvil requires characterization of the ultrasonic properties of the bond under the conditions of the experiment. Fortunately, because of the short wavelength and the large bandwidth, $\mathrm{GHz}$ interferometry offers a unique opportunity to directly 
characterize thin bonds in tcrms of their two way travel time and their attenuation [18]. This study is not only needed for our present development but will also benefit all ultrasonic studies in the $\mathrm{MHz}$ range where reproducible and well characterized bonds are essential but in which frequency range bond characterization is not possible.

The limited angle of access for $x$-rays could be improved by increasing the gasket hole dimensions relative to those of the crystal or by using gasket materials that absorb less $\mathrm{x}$-ray intensity. Another possibility is to use synchrotron radiation so that the energy can be selected to take advantage of the range of access angles.

A better method for generating a strong shear-wave signal is under development and will be needed if measurements sufficient to calculate bulk modulus are to be made.

\section{Acknowledgments}

We wish to express our thanks to G. Herrmannsdoerfer, $H$. Schultze and R. Weigel of the Bayerisches Geoinstitut for their extensive technical help with this project. We gratefully acknowledge the support of NATO in the form of a collaborative research grant to RJA and WAB, and the Alexander-von-Humboldt Stiftung for a Humboldt Preis to HS.

\section{References}

[1] F.R. Boyd and J.L. England, J. Geophys. Res. 65, 741 (1960).

[2] G.C. Kennedy and P.N. LaMori, J. Geophys. Res. 67, 851 (1962).

[3] D.L. Decker, J. Appl. Phys. 42, 3239 (1971).

[4] H. K. Mao, P.M. Bell, J.W. Shaner, D.J. Steinberg, J. Appl. Phys. 49, 3276 (1978).
[5] H. K. Mao, J. Xu and P.M. Bell, J. Geophys. Res. 91, 4673 (1986)

[6] D. Schiferl, J.N. Fritz, A.I. Katz, M. Schafer, E.F. Skelton, S.B. Qadri, L.C. Ming, M.H. Manghnani, in High-Pressure Research in Mineral Physics edited by M.H. Manghnani \& Y. Syono (Terra Sci./AGU, Tokyo/Washington, DC, 1987), p. 75. [7] A. Yoneda, H. Spetzler and I. Getting, Proc. AIRAPT/APS High Pres. Tech. Conf., 1609 (1993).

[8] A.L. Ruoff, R.C. Lincoln and Y.C. Chen, Appl. Phys. Letts. 22, 310 (1973).

[9] W.A. Bassett, A.H. Shen and M Bucknum, Rev. Sci. Instr. 64, 2340 (1993).

[10] H. Spetzler, A.H. Shen, G. Chen, G. Herrmannsdoerfer, H. Schulze and R. Weigel, Phys. Earth Planet. Int., in press.

[11] A.H. Shen, H.-J. Reichmann, G. Chen, R.J. Angel, W.A. Bassett, H. Spetzler, High Pressure Research in Geophysics, edited by M.H. Manghnani, in press.

[12] J. Koepke, W. Dieterich, J. Glinnemann, and H. Schulz, Rev. Sci. Instr. 56, 2119 (1985).

[13] H.E. King and L.W. Finger, J. Appl. Cryst. 12, 374 (1979).

[14] R.J. Angel, D.R. Allan, R. Miletich and L.W. Finger, $J$. Appl. Cryst., submitted, (1997).

[15] R.L. Ralph and L.W. Finger J. Appl. Cryst. 15, 537 (1982).

[16] I. Jackson and H. Niesler, in High-Pressure Research in Geophysics, edited by S. Akimoto and M.H. Manghnani (Center for Academic Publishing, Tokyo, 1982) p. 93.

[17] D.R. Allan, R. Miletich and R.J. Angel, Rev. Sci. Instr. 67, 840 (1996).

[18] H. Spetzler, G. Chen, S. Whitehead and I. Getting, PAGEOPH 141, 341 (1993). 